\title{
Hybrid GaN LED/elastomer membrane for uniform area illumination
}

\author{
F. Farrell ${ }^{1 *}$, E. Xie ${ }^{1}$, B. Guilhabert ${ }^{1}$, A-M. Haughey ${ }^{2}$, P. Connolly ${ }^{3}$, M. D. Dawson ${ }^{1}$ and N. Laurand ${ }^{1}$ \\ ${ }^{1}$ Institute of Photonics, Dept of Physics, SUPA, University of Strathclyde, Glasgow, UK \\ ${ }^{2}$ Fraunhofer Centre for Applied Photonics, 99 George St, Glasgow G1 1RD, UK \\ ${ }^{3}$ Department of Biomedical Engineering, University of Strathclyde, 106 Rottenrow, Glasgow, G4 0NW, UK \\ *Corresponding authors: francesca.farrell@strath.ac.uk
}

\begin{abstract}
A mechanically-flexible device for uniform area illumination is presented. The device consists of a 1 mm-thick elastomeric membrane edge-lit by a GaN LED. Homogenous irradiance above $0.13 \mathrm{~mW} / \mathrm{cm}^{2}$ at $450 \mathrm{~nm}$ over a $2.5 \mathrm{~cm}^{2}$ area is reported. Performance improvements, scalability and operation at other wavelengths are discussed.
\end{abstract}

Index Terms - Semiconductor nanocrystal, GaN, LED

\section{INTRODUCTION}

Mechanically-flexible and wearable photonics have many applications including in novel forms of displays, illuminating safety clothing, and in health technology [1,2]. An example of the latter is phototherapy where UV or visible light is used to treat skin disorders such as psoriasis, eczema and vitiligo [3]. While phototherapy is typically carried out in a clinical environment with bulky lamp systems, GaN-based LEDs are now facilitating the emergence of at-home devices to improve patient convenience and decrease demands on the healthcare system [4]. However, GaN LEDs are inherently point sources and current at-home devices consist of rigid LED arrays that produces non-uniform light distribution over the treatment area, in turn limiting efficacy [3]. These devices are also not mechanically flexible and therefore not truly wearable. OLEDs could be a solution to these issues, however they suffer from limited brightness, efficiency and wavelength scalability (in particular in the blue and UV range) compared to inorganic LEDs.

Herein, we propose to combine custom formats of mini- and micro-size GaN LEDs ( $\mu$ LEDs) with a mm or sub-mm-thick polydimethylsiloxane (PDMS) membrane in an edge-lit configuration (Fig. 1a) The PDMS sheet acts as a waveguide to deliver the LED light to the illumination area. It is biocompatible, transparent from $290 \mathrm{~nm}$ upwards, and is an effective waveguide material [5]. Optionally, a colorconverting material can be easily included for applications requiring operation at another wavelength or for multiwavelength operation.

In the following, we focus on the characterization of the light delivery and diffusing membrane for a $2.5 \mathrm{~cm}^{2}$ illumination area. The diffusing region is obtained by adding on top of the light delivery sheet either (i) a PDMS structure (flat or tapered) with embedded $\mathrm{TiO}_{2}$ scattering particles for extraction and diffusion of the LED light through the top surface or (ii) a PDMS sheet with embedded colloidal quantum dots (CQDs) for

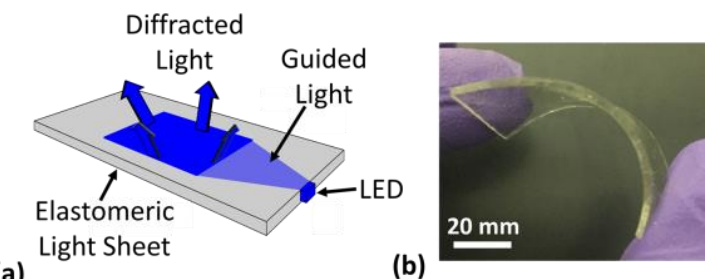

(a) Light Sheet (igh

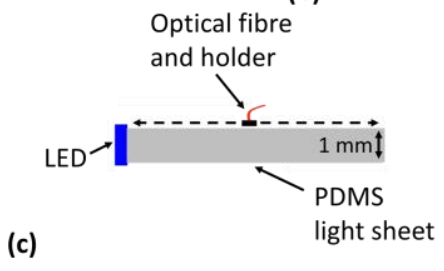

Figure 1 - (a) device schematic; (b) fabricated PDMS light sheet showing flexibility; (c) schematic of the irradiance measurements setup.

color-conversion. Scalability and performance improvements are discussed.

\section{Materials AND Methodology}

The PDMS light sheet for this study is $1 \mathrm{~mm}$ thick with dimensions of $40 \times 20 \mathrm{~mm}^{2}$ (Fig. 1b). It is fabricated using a ratio of 5:1 silicon to cross linker, and cured at room temperature for 48 hours. A commercial broad area GaN LED with an optical power of $100 \mathrm{~mW}$ and a peak emission wavelength of $450 \mathrm{~nm}$ is butt-coupled to this light sheet in an edge-lit configuration for the initial optical characterization. The diffused light from the top surface of the light sheet is measured as irradiance $\left(\mu \mathrm{W} / \mathrm{cm}^{2}\right)$ using an optical fiber and a calibrated spectrometer (Fig. 1c). Translating the optical fiber enables irradiance measurements along one spatial direction. For 2D irradiance mapping, a CCD and lens system are placed above the setup and calibrated. The flat diffusing structure ( 15 $\mathrm{mm} \times 15 \mathrm{~mm} \times 1 \mathrm{~mm})$ is produced by mixing $\mathrm{TiO}_{2}$ nanoparticles (25 nm mean diameter) with PDMS at a weight ratio of $0.05 \%$ and cured as before. The $15^{\circ}$ tapered diffusing structure is fabricated similarly and has a maximum thickness of $4 \mathrm{~mm}$. For the color-converting structure $(10 \mathrm{~mm} \times 10 \mathrm{~mm} x$ $1 \mathrm{~mm})$, red emitting CdSSe/ZnS CQDs $(630 \mathrm{~nm})$ are mixed in PDMS at a weight ratio of $0.04 \%$ CQD to PDMS cured at room temperature for 72 hours. These scattering/diffusing or colorconverting structures are then placed directly on the PDMS light sheet, $15 \mathrm{~mm}$ from the edge. 
(a)
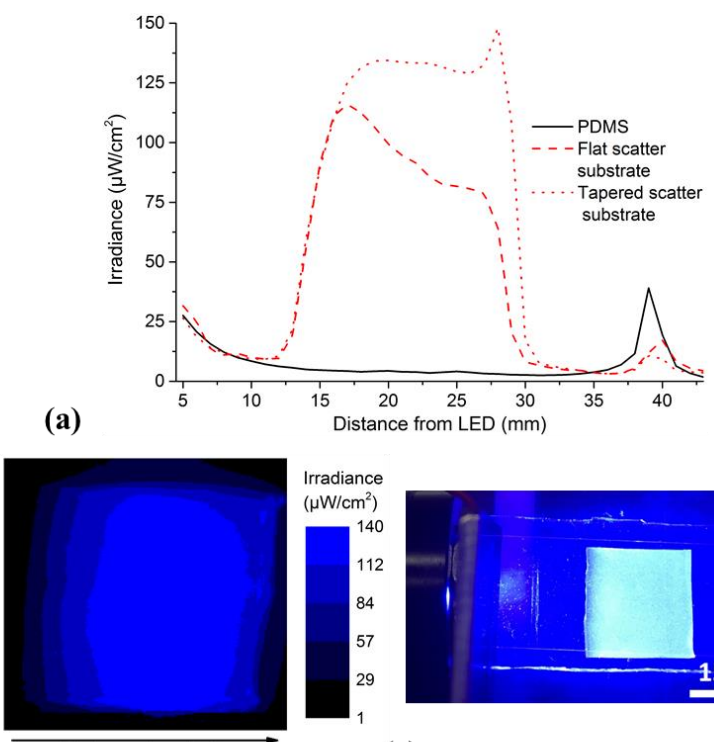

(b)

Distance from LED

Figure 2 - (a) Irradiance as a function of distance from the LED for the PDMS light sheet without and with the scattering structure; (b) $2 D$ irradiance map; $(c)$ image of the sheet with LED switched on - the scattering region is clearly identified.

\section{RESULTS AND DISCUSSION}

Fig. 2a plots the 1D irradiance of a device for $450 \mathrm{~nm}$ illumination with the flat and tapered scattering structures. Data for a device with no diffusing structure is also given as a reference. The irradiance of the PDMS reference sample decreases with distance from the LED, excluding the end of the membrane $(40 \mathrm{~mm})$ where edge effect occurs. The initial decrease with distance corresponds to light that is not guided in the PDMS sheet (leaky modes). At longer distances, the irradiance comes from scattered guided light. The addition of the flat scattering substrate (dashed line) greatly increases the irradiance over the illumination area with a maximum of $115 \mu \mathrm{W} / \mathrm{cm}^{2}$, a 22 -fold increase compared to the reference sample. However, the irradiance is not uniform over the illumination area. One method of improving this uniformity is to use the tapered substrate with increasing scattering particles as the LED distance increases. This technique is effective in producing uniform $( \pm 4 \%)$ irradiance of $130 \mu \mathrm{W} / \mathrm{cm}^{2}$. In order to see this effect across both the $\mathrm{x}$ and $\mathrm{y}$ directions, an image was taken of the full scattering substrate and the image data analyzed to produce a map of irradiance over the scattering substrate (Fig. 2b). As can be seen, the irradiance remains constant over the width of the scattering substrate, providing the information that uniform irradiance can be achieved over an area of $2.5 \mathrm{~cm}^{2}$. The downside of the tapered structure is that it increases the total thickness of the device to $5 \mathrm{~mm}$. Alternatives that would minimize device thickness include injecting light from both ends of the membrane or using a spatially graded $\mathrm{TiO}_{2}$ spheres concentration.

Fig. 3a shows data for 1D irradiance plot when the colorconverting structure is used in place of the scattering samples. The reference PDMS sample is again plotted. Up to $150 \mu \mathrm{W} / \mathrm{cm}^{2}$ irradiance at the color-converted wavelength is

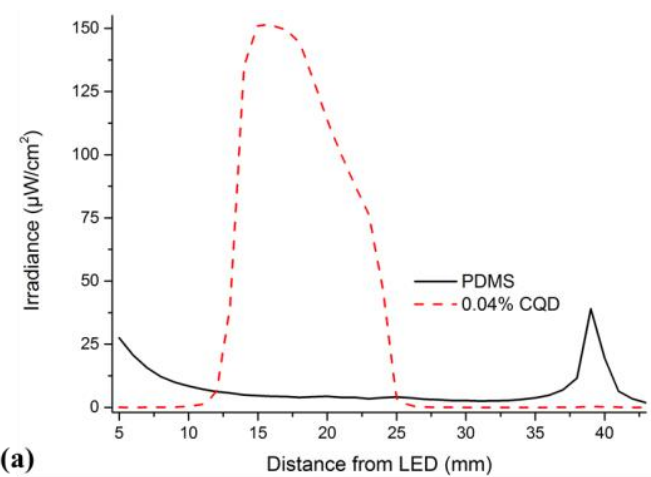

(b)

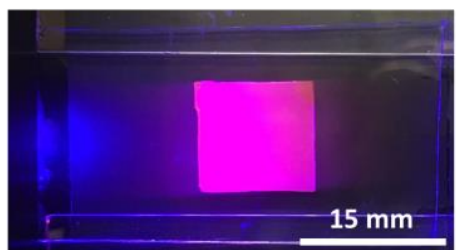

Figure 3 - (a) Irradiance trend with distance from the LED of CQD sample; (b) image of the PDMS membrane with a CQD sample.

achieved. Fig. $3 b$ is an image of the device showing the colorconverted light over the illumination area. This option is attractive for multi-wavelength phototherapy applications.

The results shown above utilized a GaN LED with an emission size of $3 \times 3 \mathrm{~mm}^{2}$, larger than the light sheet thickness. Therefore, the LED light coupling efficiency is low. A custommade $\mu$ LED array will allow for an increased coupling efficiency and can be designed in a way to minimize the source thickness whilst increasing the length, providing more light overall to the PDMS light sheet. Modeling and initial results with $\mu$ LED integration will be shown and discussed.

\section{CONCLUSIONS}

In this work, we have demonstrated a mechanically flexible light emitting device which has potential applications in displays and health technology. We coupled a GaN LED to a PDMS elastomeric light sheet and reported the effects of using $\mathrm{TiO}_{2}$ nanoparticles and CQD color-converters encapsulated in PDMS. An irradiance value of $130 \mu \mathrm{W} / \mathrm{cm}^{2}$ was achieved at $450 \mathrm{~nm}$ over an area of $1.5 \mathrm{~cm}^{2}$.

\section{ACKNOWLEDGMENTS}

This work was supported by the EPSRC Grant EP/L015595/1. Supporting data is available:

DOI: http://dx.doi.org/10.15129/0a87873f-b9d1-414f-aa41f07a184e6ae5

\section{REFERENCES}

[1] J. Hu et al, Optical Materials Express, 2013, 3, 1313-1331.

[2] B M Qaundt et al, Biomedical Optics Express, 2017, 8, 4316-4330.

[3] C. Cochrane et al, Mater Sci Eng C Mater Biol Appl, 2013, 33, 11701175 .

[4] S. Lim, Journal of the SID, 2011, 19, 882-887.

[5] J-H. Lee et al, Journal of the SID, 2008, 16, 329-335. 